\title{
1-D Acoustical Analysis of Axial-Inlet, Transverse- Outlet Air Cleaners of Rectangular and Circular Cross-Section
}

\author{
M. L. Munjal and N. K. Mukherjee \\ Facility for Research in Technical Acoustics (FRITA), Department of Mechanical Engineering, Indian Institute of \\ Science, Bangalore-560 012, India
}

\begin{abstract}
This paper deals with the prediction of the acoustical performance of two basic types of air cleaners: 1) rectangular axial-inlet transverse-outlet (RAITO) and 2) circular axial-inlet transverse-outlet (CAITO), through 1-D acoustical analysis. Predictions of the acoustical performance of the air cleaners have been validated with 3-D FEM results. The acoustical performance of the cleaner box has been compared with the bare plenum performance (without any filter element) in order to estimate the acoustical performance of the filter element. It has been shown that even if inlet and outlet pipes do not lie exactly on the centre of the corresponding cross section, the 1-D formulation still can predict the transmission loos (TL) with reasonable accuracy in the low- and mid-frequency zones. The 1-D model presented here is not only more convenient and much faster than the 3-D model, and it has the additional advantage of being able to incorporate the effect of mean flow, particularly the dissipative effect of mean flow at the inlet/outlet of the air cleaner. This 1-D model would facilitate a rational design of an air cleaner for control of the intake noise of internal-combustion engines.
\end{abstract}

\section{INTRODUCTION}

An air cleaner is an element that is used to purify air by removing dust particles, bacteria, and more from the air. Air cleaners can be used for internal combustion engines, cabin air filtration, or ventilation systems. Here, we concentrate on the acoustical performance of air cleaners used in the intake systems of the internal combustion engines for reducing mechanical wear inside the engine. Though there are several configurations of the cleaner box available on the market, in our present analysis we concentrate on a simple rectangular air cleaner that uses paper as the filtering element. We are going to focus mainly on the following two configurations:

1. rectangular axial-inlet transverse-outlet (RAICO) cleaner

2. circular axial-inlet transverse-outlet (CAICO) cleaner

Figure 1 shows how an air filter element looks when removed from the cleaner box. The flow enters the filter in a plane perpendicular to the paper.

Some of the relevant investigations reported in the literature are as follows. Hao et al. gave a virtual design and performance prediction of a silencing air cleaner used in an internal combustion engine intake system. ${ }^{1}$ They gave an analysis of the air cleaner through the Boundary Element Method. Knutsson et al. tried to take into account the acoustically nonrigid nature of an air cleaner box (generally made of plastic). ${ }^{2}$ They found that the flow-related losses dominate at lower frequencies, whereas losses due to the acoustically non-rigid nature of the walls dominate at higher frequencies. Sim et al. proposed an optimal design scheme to reduce the noise of the engine intake system through support vector regression techniques. ${ }^{3}$ The length and radius of all the components in the engine intake assembly were given as input, and their optimum dimensions were found through simulation using a noiseminimization criterion. Chen et al. developed a numerical model to optimize filter design. ${ }^{4}$ In their analysis, a generalized correlation curve was found for different filter media, using non-dimensional parameter analysis. The analysis showed that optimal pleat count increases with decreasing filter permeability. Fabbro et al. generated a dimensionless model to estimate the pressure drop across clean-pleated filters. ${ }^{5}$ The model was derived from the results obtained using high-efficiency and low-efficiency particulate filters. According to this analysis, air flow distribution is homogeneous over high-efficiency filters, and initial pressure drop is greatly affected by the geometric characteristics of a low-efficiency filter.

In this paper, a one-dimensional analysis is presented to derive a transfer matrix that can be incorporated as a subroutine in the transfer matrix-based muffler software FRITAmuff ${ }^{6}$ for 1-D acoustical analysis of the entire air-intake system and the exhaust system of an internal combustion engine.

\section{RECTANGULAR AXIAL-INLET TRANS- VERSE-OUTLET (RAITO) CLEANER}

The one-dimensional acoustical analysis model proposed below makes use of an analogy to a two-duct perforated crossflow element. ${ }^{7}$ Results of $1-\mathrm{D}$ analysis are validated with those from the 3-D finite element software SYSNOISE. ${ }^{8}$

\subsection{General Assumptions}

The analytical model developed here is based on the following assumptions:

i All of the following models are based on the fact that the transverse dimensions of the air cleaner are considerably small compared to the axial dimension. It may not be true for all filter configurations, but this assumption will still hold well up to certain range of frequency. 